\title{
Bronchobiliary fistula due to acute cholecystitis in a suprahepatic gall bladder
}

\author{
M.C. Allison, S. Milkins, A.K. Burroughs, H.S. Rogers and H.C. Thomas \\ Academic Departments of Medicine, Histopathology and Surgery, Royal Free Hospital and School of Medicine, \\ London, NW3 2QG, UK.
}

\begin{abstract}
Summary: We describe a patient presenting with painless jaundice, anorexia and pruritus. The gall bladder was found to be lying above and behind a hypoplastic right lobe of liver. There was no evidence of cholangitis or biliary obstruction. The patient subsequently developed a bronchobiliary fistula with severe wheeze, cough and bile-stained sputum. Emergency percutaneous drainage of the gall bladder led to immediate cessation of bronchospasm and biloptysis, rendering the patient fit for definitive surgery.
\end{abstract}

\section{Introduction}

Expectoration of bile is pathognomonic of a bronchobiliary fistula. ${ }^{1}$ This is rare and usually associated with intrahepatic abscess or hydatid disease. ${ }^{2}$ Less common causes are congenital fistulae, trauma or surgical manoeuvres. ${ }^{1}$ A few cases have been described in association with obstruction of the biliary tree due to calculi, ${ }^{3}$ post-operative biliary stricture, ${ }^{4}$ or chronic pancreatitis. ${ }^{5}$ Bronchobiliary and bronchopleural fistulae may also result from acute cholecystitis complicated by gall bladder perforation and subphrenic abscess. ${ }^{1,4}$

We report a patient who was initially thought to have acute hepatitis followed by cholestasis and was found to have congenital abnormalities of the liver and biliary tree. He subsequently developed acute cholecystitis complicated by subphrenic abscess and bronchobiliary fistula. Immediate percutaneous gall bladder drainage was life-saving, allowing improvement of the patient's condition prior to surgery.

\section{Case report}

A 63 year-old man presented with a two week history of anorexia, pruritus, pale stools and dark urine. He had been an insulin-dependent diabetic for 10 years but was on no other medication. On examination he was markedly jaundiced with scratch marks. A firm smooth non-tender liver was palpable $12 \mathrm{~cm}$ below the

Correspondence: M.C. Allison M.B., M.R.C.P. Academic Department of Medicine, Royal Free Hospital, Pond Street, London, NW3 2QG, UK.

Accepted: 21 October 1986 costal margin, and the spleen was just palpable. He had a serum bilirubin of $173 \mu \mathrm{mol} / 1$ (normal $<$ $17 \mu \mathrm{mol} / \mathrm{l}$ ), an alkaline phosphatase of $827 \mathrm{IU} / 1$ (nor$\mathrm{mal}<130 \mathrm{IU} / \mathrm{l})$ and aspartate transaminase (AST) of $2085 \mathrm{IU} / 1$ (normal < $40 \mathrm{IU} / 1$ ). Serum albumin was normal but the prothrombin time was prolonged at 21 seconds, correcting to 14 seconds after vitamin $\mathrm{K}$. Autoantibodies and serological markers for viral hepatitis were absent.

Ultrasound failed to identify the extrahepatic biliary tree or gall bladder, but the intrahepatic bile ducts were not dilated. Liver biopsy showed abundant fibrous septa, centred around portal areas, intersecting the tissue and in places forming nodules. Bile duct proliferation, neutrophilic infiltrates and hepatocellular injury with Mallory bodies were striking features, and there was excessive copper associated protein. The overall appearances, while unusual and not diagnostic, suggested chronic biliary tract disease. Percutaneous cholangiography showed a common bile duct of normal calibre and free flow of contrast into the duodenum.

While being investigated, his serum bilirubin rose to $452 \mathrm{IU} / 1$, and alkaline phosphatase remained high at $601 \mathrm{IU} / 1$. The AST had fallen to $153 \mathrm{IU} / \mathrm{l}$. This biochemical pattern suggested an acute hepatocellular injury followed by cholestasis, perhaps due to non-A non-B hepatitis. He was therefore given cholestyramine and a therapeutic trial of prednisolone $30 \mathrm{mg} /$ day. ${ }^{6}$ His pruritus resolved rapidly and his liver function tests improved with a fall in bilirubin to $20 \mu \mathrm{mol} / 1$, although the alkaline phosphatase remained elevated at $417 \mathrm{IU} / 1$.

The prednisolone was tailed off 9 months later. Soon afterwards his jaundice and pruritus returned 
and he was readmitted for further investigation. Liver biopsy appearances were unchanged. A computerized tomographic (CT) scan without intravenous contrast (Figures 1 and 2) showed an irregular liver outline and a large lucent area in the posterior part of the right lobe which was thought to be a benign simple cyst. Endoscopic retrograde cholangiography (ERC) revealed a large gall bladder lying in an unusual position high up in the right lobe of liver (Figure 3). Repeat CT scanning immediately after ERC confirmed that the liver 'cyst' filled with contrast and was, in fact, an ectopic gall bladder.

He was readmitted 3 months later with right hypochondrial pain, increasing pruritus, cough and green sputum. Portosystemic encephalopathy and ascites had developed. He was found to have marked bronchospasm and a chest X-ray showed collapse and consolidation of the right lobe. Despite antibiotics, bronchodilators and chest physiotherapy, his bronchospasm worsened and his cough became productive of bile (up to $100 \mathrm{ml} /$ day). A percutaneous drain was inserted into the gall bladder under CT control with an immediate reduction in bronchospasm and no further bile expectoration. ERC demonstrated a communication between the gall bladder and a cavity above the liver posteriorly.

At operation an abscess cavity was found in communication with the gall bladder and diaphragm. The right lower lobe of lung was adherent to the diaphragm and a fistula was identified (Figure 4). The right lobe of liver was hypoplastic and the left lobe enlarged. The abscess cavity was drained and the diaphragmatic defect sutured. The gall bladder was freed and

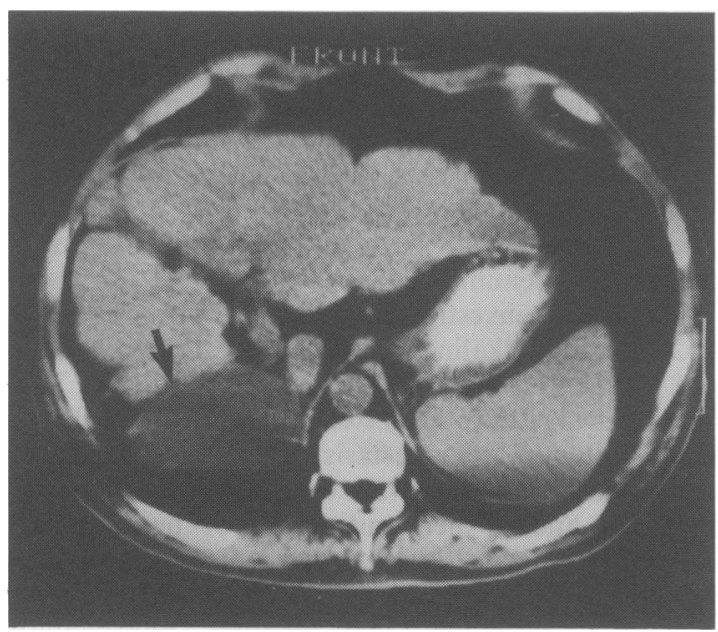

Figure 1 CT scan showing liver with an irregular outline suggesting cirrhosis, and an apparent cyst posteriorly (arrowed). removed from within the right lobe of liver. An operative cholangiogram via the cystic duct showed气 free flow of contrast into the duodenum. Histology of $\frac{3}{\infty}$

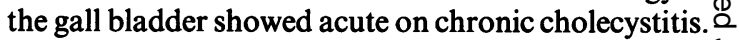

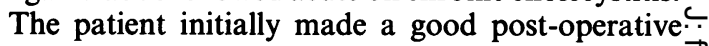
recovery but then developed worsening ascites and $\overrightarrow{\vec{F}}$ renal function. He died 4 weeks post-operatively fromcombined hepatic and renal failure. At post-mortem $\frac{C}{O}$ the liver weighed $1300 \mathrm{~g}$ and showed reversal of the $\overline{\bar{c}}$. normal relationship between lobe size. The gall blad- $\widehat{\nabla}$ der bed was identified on the posterior aspect of the right lobe. The site of ligature of the cystic duct was ${ }^{\infty}$ identified, and the common bile duct and each hepatic $\vec{\circ}$ duct appeared to conform to the normal anatomy of $\overrightarrow{-}$ the porta hepatis. The vascular radicles supplying the ${ }_{\sigma}^{\omega}$ liver also appeared normal. No evidence of congenitalo abnormality was seen in the rest of the body. Sections 3 from both lobes and hila showed identical features and were characterized by portal fibrosis with nodule $\omega$ formation.

\section{Discussion}

This case displayed a confusing combination of clin- $\vec{D}$ ical, radiological and histopathological features. The patient's initial presentation resembled an acute $\vec{\circ}$ hepatitis with anorexia, painless jaundice and extremely high aspartate transaminase leve However, the correction of the prothrombin time wif vitamin $\mathrm{K}$, and pruritus, implied cholestasis. Livêt biopsy supported chronic biliary tract disease However percutaneous cholangiography did not iden-

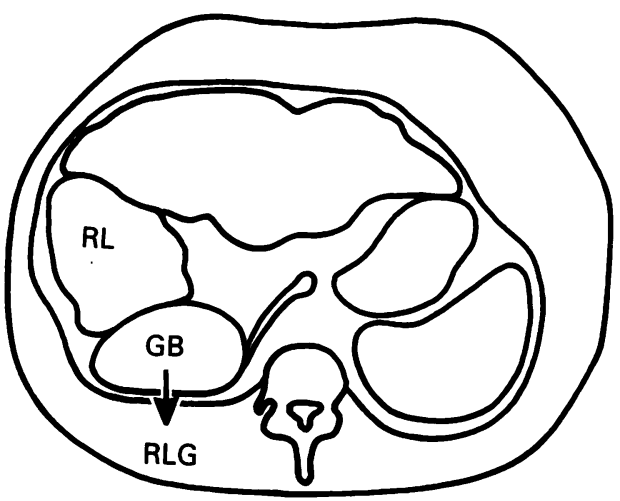

Figure 2 Line diagram illustrating the features seen on the CT scan in Figure 1. The gall bladder (GB) is lying behind the hypoplastic right lobe of liver (RL) and anterior to the diaphragm. The arrow shows the direction of the subsequent fistula between the acutely inflamed gall bladder and the right lung (RLG). 


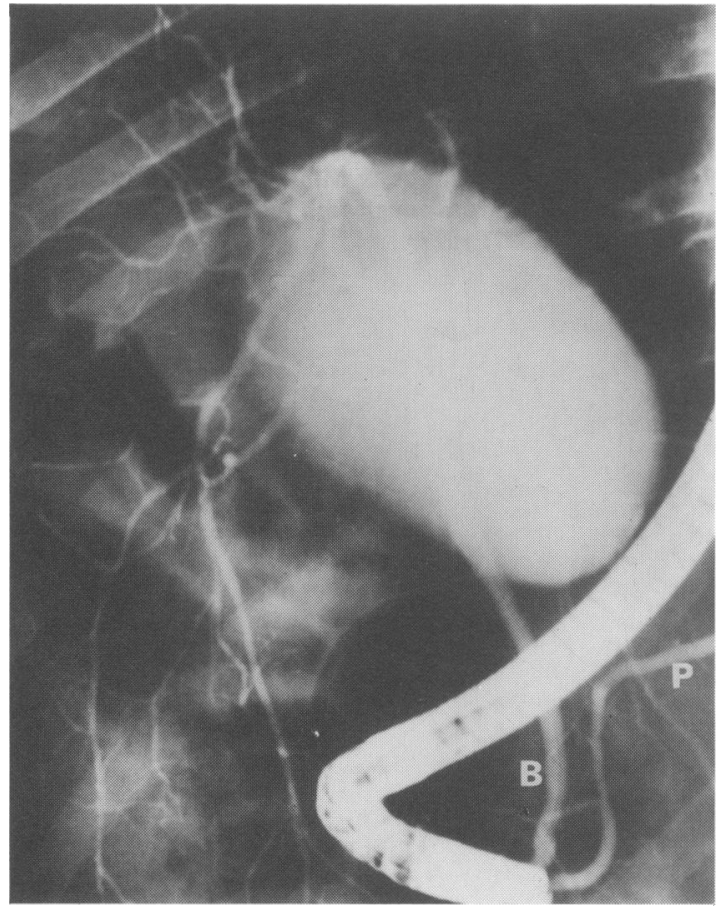

Figure 3 ERCP showing the pancreatic duct $(P)$, the common bile duct (B) intrahepatic ducts of normal calibre, and the abnormally positioned gall bladder.

tify any primary bile duct disease or extrahepatic obstruction.

We believe the most likely explanation for the initial high AST level and subsequent hepatocellular dysfunction was recurrent cholecystitis superimposed on the congenital abnormality. A transiently high AST level (600) may be associated with extrahepatic biliary disease. $^{7}$ The abnormal gall bladder location and the use of corticosteroids may have masked symptoms of the cholecystitis and biliary sepsis. There are four previous cases described with a hypoplastic right lobe of liver and abnormal positioning of gall bladder and cystic duct. ${ }^{8,9}$ Acute cholecystitis developed in two of these patients but neither was complicated by chronic cholestasis or bronchobiliary fistula.

It is of interest that bronchobiliary fistula presented

\section{References}

1. Saylam, A., Ersoy, V., Boris, I. et al. Thoracobiliary fistulas: report of six cases. Br J Dis Chest 1974, 68: 264272.

2. Amir-Jahed, A.K., Sadrieh, M., Farpour, A. et al. A surgical complication of hepatic echinococcosis and amebiasis. Ann Thorac Surg 1972, 14: 198-204.

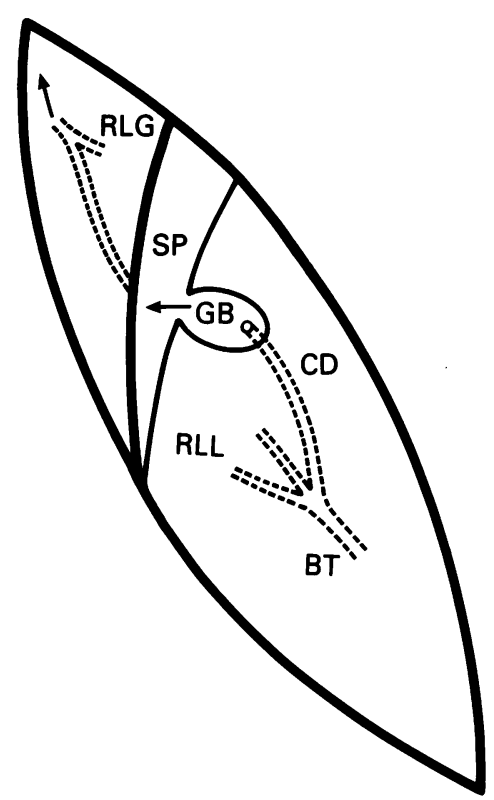

POST.

ANT.

Figure 4 Diagram of the operative findings showing the fistula between the suprahepatic gall bladder (GB), subphrenic abscess cavity (SP) and right lung (RLG). The positions of the right lobe of liver (RLL), cystic duct (CD) and biliary tree (BT) are shown.

with cough and green sputum in our patient; this experience has been reported previously. ${ }^{10}$ Chest physiotherapy may have precipitated the opening of the fistula and the sudden deterioration in our patient. Emergency percutaneous drainage of the suprahepatic gall bladder under CT control stopped the flow of bile into the bronchial tree, relieved the bronchospasm, and rendered the patient fit for surgical intervention.

\section{Acknowledgements}

We thank Professor P.J. Scheuer for his help in the interpretation of the liver histology, Professor K.E.F. Hobbs who performed the operation, and Dr R. Dick who performed the percutaneous drainage of the gall bladder bed.

3. Wei, W.I., Choi, T.K., Wong, J. \& Ong, G.B. Bronchobiliary fistula due to stones in the biliary tree: report of two cases. World J Surg 1982, 6: 782-785.

4. Boyd, D.P. Bronchobiliary and bronchopleural fistulas. Ann Thorac Surg 1977, 24: 481-487.

5. Walters, D.A.K., Barker, E.M. \& Kallideen, J.M. Bron- 
chobiliary fistula after chronic pancreatitis. $S$ Afr Med J 1984, 6: 576-577.

6. Sherlock, S. Virus hepatitis. In: Diseases of the Liver and Biliary System, Seventh edition. Blackwell Scientific Publications, Oxford, 1985, pp 251-279.

7. Fortson, W.C., Tedesco, F.J., Starnes, E.C. \& Shaw, C.T. Marked elevation of serum transaminase activity associated with extrahepatic biliary disease. J Clin Gastroenterol 1985, 7: 502-505.
8. Faintuch, J., Machado, M.C.C. \& Raia, A.A. Suprahepatic gall bladder with hypoplasia of the right lobe of the liver. Arch Surg 1980, 115: 658-659.

9. Youngwirth, L.D., Peters, J.C. \& Perry, M.C. The supra- $\subset$ hepatic gall bladder. Radiology 1983, 149: 57-58.

10. Pappas, S.C., Sasaki, A. \& Minuk, G.Y. Bronchobiliary fistula presenting as cough with yellow sputum. $N$ Engl $\overline{{ }_{C}}$ Med 1982, 307: 1027. 